\title{
The equilibrium of cloud manufacturing system under cost uncertainty
}

\author{
Wei GUO*, Wei PENG* and Lei WANG* \\ * Key Laboratory of Mechanism Theory and Equipment Design of Ministry of Education, Tianjin University \\ 135 Yaguan Road, Haihe Education Park, Jinnan District, Tianjin, 300350, China \\ E-mail: tjuwl@tju.edu.cn
}

Received: 13 January 2016; Revised: 20 January 2017; Accepted: 9 May 2017

\begin{abstract}
In this paper, we explore the equilibrium problem of cloud manufacturing system (CMfgS) with cost uncertainty. We propose a CMfgS supernetwork model in which decision-makers (resource service providers (RSPs), the agent and resource service demanders (RSDs)) seek to determine their optimal behavior in an environment with cost uncertainty. The cost uncertainty is represented by random variables and the decision-makers' risk aversion attitude is modeled by adopting mean-variance utility. The equilibrium conditions governing the supernetwork model are proposed based on variational inequalities. To investigate the impacts of decision-makers' risk aversion attitude and the degree of cost uncertainty on equilibriu m of CMfgS, we conduct a numerical analysis in this study. The results indicate that a risk averse RSP (or agent) tends to raise selling price to mitigate risk, and a risk averse RSD is willing to pay more for the needed services. We also show that a risk neutral RSP could sell more services when the variance of cost distribution is relative large but the opposite occurs for risk averse RSPs.
\end{abstract}

Key words : Cloud manufacturing system, Equilibrium, Cost uncertainty, Risk aversion, Variational inequalities

\section{Introduction}

Cloud manufacturing (CMfg), which was proposed in 2010, is an emerging service oriented manufacturing mode. The core idea of CMfg is manufacturing as a service, and the aim of it is to realize the free sharing, high utilization, and on-demand use of manufacturing resources and capabilities through providing reliable, on-demand, high quality, cost effective manufacturing services in a cloud manufacturing system(CMfgS) (Tao et al., 2011).

In $\mathrm{CMfgS}$, there are three kinds of roles involved in service transactions: resource service provider (RSP), resource service demander (RSD), and the agent (also called as cloud operator). The RSPs own the manufacturing resources and abilities which are provided to the agent on demand. The RSDs are service consumers. They submit service requirements to the agent and gain optimal services from it. The agent is responsible for searching, combining, coordinating and managing the required services for meeting RSDs' requirements. After a RSD's request is received by the agent, it searches in the database and propose a reliable and optimal service scheme for the RSD. When the RSD modifies and confirms the service plan, the agent sends service requests to the selected RSPs. then specific services are organized and launched to meet the RSD's needs. Finally, the service output is sent to the RSD (Wang and Xu, 2013). In this process, each participant is an independent decision-maker. CMfg S has the advantage of managing dynamic service resources in a systematic and integrated way and providing reliable, on-demand, high quality services for RSDs. With the help of the agent's intelligent management, RSDS need not take much time to select suitable services and design service combination schemes. Though RSPs and RSDs must pay for the agent's management, they can also save much time and money in the process of transaction.

A substantial literature has grown up around service transactions of CMfgS in recent years. Most of these articles mainly focus on transaction process management, such as cost management (e.g., Cheng et al., 2011), transaction process monitoring (e.g., Morgan and O’Donnell, 2015), service optimal allocation (e.g., Laili et al., 2012), etc. while 
little attention has been paid to benefit equilibrium in CMfgS. In fact, in service transaction process, each participator makes decisions from $\mathrm{h}$ is/her own perspective. The RSDs seek to purchase the required high quality services. While the agent and the RSPs seek to maximize their profit. An important question, therefore, is whether there is an equilibrium state that every decision-maker can gain maximu m revenue. The equilib riu $\mathrm{m}$ is essential for the development of $\mathrm{CM} f g \mathrm{~S}$ since, if some participators are incapable of obtaining satisfying profit for a long time, they have incentives to leave. As a consequence of this, the users gradually drift away from the CMfgS. Therefore, it is of great importance to study the equilibrium problem of CMfgS.

As stated above, the literature on equilibrium of cloud service system is very limited. Tao et al. (2011) studied the utility equilibrium between decision-makers. They proposed a utility coordination method to improve the comprehensive utility of the resource service transaction chain under the decentralized decision-making condition. Ardagna et al. (2013) and Anselmi et al. (2014) modeled the Nash equilibrium of service provisioning problem in cloud system. Yang and Cai (2014) analyzed the Stackelberg equilibrium between cloud storage provider and end user. Mazalov et al. (2015) studied the price equilibrium of a three-level market composed of service providers, brokers and end users. Other related works can be found in the field of service-oriented network. And most of these studies explored the equilibrium between service providers of the service-oriented network from the perspective of game theory (e.g., Nagurney and Wolf, 2014; Zhang et al., 2010; Shen et al., 2007; Krishan et al., 2002). Besides, Lu (2012) analyzed the equilibrium price strategy and proposed an optimal decision model for designing service-oriented manufacturing. Sun et al. (2006) discussed the optimization of utility in service negotiation.

Although these studies have made a significant contribution to our knowledge about equilibriu metween decision makers, they have neglected the cost uncertainty. Cheng (2002) argued that cost uncertainty is a consequence of technological or environ mental uncertainty. In $\mathrm{CMfgS}$, there are many factors that would lead to cost uncertainty, such as random demand, emergencies and the degree of customization. The cost uncertainty may cause risk loss since the participators have to make decisions without full knowledge about its consequences (Askar, 2014). Another weakness in the existing literature is that most studies have potentially assumed that decision makers are risk neutral. However, some empirical studies in supply chain management suggest that decision makers' behavior are not always in accord with this hypothesis (e.g., Brown and Tang, 2006; Schweitzer and Cachon, 2000). Compared to risk neutrality, risk aversion which means a more conservative behavior in decision-making (Caliskan-Demirag, 2011) is more common in service transactions, and would lead to different outcomes. Motivated by these studies, we incorporate risk aversion attitude and cost uncertainty into the analys is of equilibrium of CMfgS.

The main aim of this study is to analyze the equilibrium conditions of CMfgS and investigate the influences of decision-makers' risk attitude and the degree of cost uncertainty on the equilibrium, and thereby contribute to the understanding of decision-makers' transactional behavior. We consider $m$ RSPs, one agent and $n$ RSDs in this paper. The agent is an intermediary and transacts with both the RSPs and the RSDs. The services are achieved in time, in high quality, without RSDs' knowledge about how and where the services are supplied (Wang and Xu, 2013). All the decision-makers seek to maximize their profits. We model cost uncertainty with a stochastic variable in the form of additive random term. And risk aversion is modeled by adopting the mean-variance utility. We state the equilibrium conditions based on variational inequalities (VI). To explore the potential impacts of risk aversion and cost uncertainty on equilibrium solutions, we conduct a numeric analysis and consider the variation of decision-makers' risk aversion attitude and variance of cost distribution. The combined effects of different parameters are analyzed in this study.

Our paper contributes to the growing literature in several ways. First, we model the relationship between decision-makers in $\mathrm{CMfgS}$ with a supernetwork, which provides a new perspective on behavior analysis of the participators in CMfgS. Second, we analyze the equilibrium conditions of CMfgS under cost uncertainty and provide the VI formu lation, which advances our knowledge of equilibrium of $\mathrm{CMfgS}$. Third, we explore the influences of risk attitude and the degree of cost uncertainty on equilibrium solutions of CMfgS. We show that A risk averse RSP (or agent) tends to raise selling price to mitigate risk, and a risk averse RSD is willing to pay more for the needed services . We also show that A risk neutral RSP could sell more services when the variance of cost distribution is relative large but the opposite occurs for risk averse RSPs. Our results fill a gap in the literature and provide a pre liminary foundation for future research.

The rest of our paper is organized as follows. In Section 2, we describe the CMfgS supermetwork model with basic parameters and present the decision-makers' optimal behavior. Section 3 states the equilibriu m conditions of CM fgS. In Section 4, we explore the influence of risk attitude and the degree of cost uncertainty on equilibrium solutions. In 
Section 5, we conclude the paper and present extension ideas.

\section{Model analysis}

\subsection{The CMfgS supernetwork model}

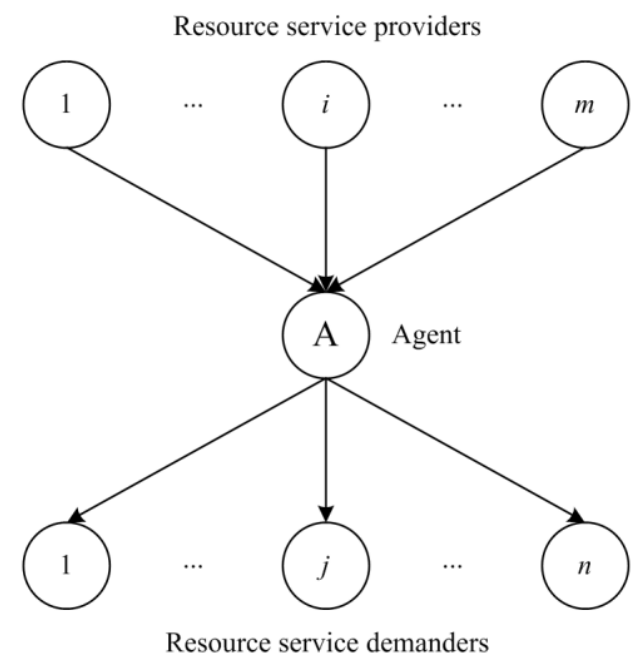

Fig. 1 The structure of CMfgS supernetwork model.

We use a supernetwork model to illustrate the relationship between decision-makers of CMfg S. As depicted in Fig. 1 , the supernetwork consists of three tiers. The top-tier nodes represent $m$ RSPs who are involved in offering manufacturing services. They sell the services to the agent in the second tier. We assume that the RSPs compete with one another in a non-cooperative manner. They need to cover the service costs and the transaction costs and decide what the price should be set and how many services should be supplied.

Agent, which is represented by the second-tier node in Fig. 1, functions as the intermediary. In fact, there are three agents in CMfgS: interface agent, broker agent and supervision agent (Wang and Xu, 2013). They work cooperatively to provide high quality and effective intermediary service for RSPs and RSDs. Since there is no competition among these agents, they can be viewed as an integrated whole. The agent transact with RSPs and RSDs, respectively. In an ideal system, the agent is smart enough to deal with the service transactions (Wang and Xu, 2013). We assume that the agent needs to cover the transaction costs and determine the selling prices and the quantity of the services sold to RSDs in order to maximize its profit.

The bottom-tier nodes in Fig. 1 represent $n$ RSDs. They transact with the agent to obtain the services needed. We assume that each RSD has an elastic demand, which means that the demand for services depends on other factors like price, quality, etc. The RSDs need to cover the transaction costs and decide the demand as well as the reserve prices.

We denote a typical RSP by $i$, and a typical RSD by $j$. For completeness, we first describe the behavior of the decision-makers, and then state the equilibrium conditions of a CMfgS. Subsequently, we analyze the impact of risk uncertainty and cost uncertainty on equilibrium of CMfgS numerically. The majority of the needed notation is given in Table 1.

\subsection{The optimal behavior of decision-makers in CMfgS}

In this section, we used three interconnected utility models to describe the optimal behavior and the optimality conditions of RSPs, the agent and RSDs in CMfgS, respectively. The RSPs' and the agent's utility were described in the first two models which considered the decision-makers' net profits. The third model formulated the RSDs' utilities by establishing the link between reserve payments and actual pay ments. The decision-makers' risk attitude is incorporated into the three models that are indispensable to the equilibrium analysis of CMfgS.

\subsubsection{The behavior of RSPs}


Table 1 Notation used in this paper

\begin{tabular}{|c|c|}
\hline Notation & Definition \\
\hline$s_{i}$ & The quantity of services provided by $R S P_{i}$ to the agent \\
\hline$S^{1}$ & Vector of the quantity of services supplied by RSPs with component: $s_{i}, \ldots, s_{m}$ \\
\hline$s_{j}$ & The quantity of services sold by the agent to $R S D_{j}$ \\
\hline$S^{2}$ & Vector of the quantity of services provided by the agent with component: $s_{j}, \ldots, s_{n}$ \\
\hline$\overline{S_{i}}$ & Maximum service capacity of $R S P_{i}$ \\
\hline$f_{i}\left(s_{i}, \varepsilon_{i}\right)$ & Service cost function of $R S P_{i}$ \\
\hline$c_{i}\left(s_{i}, \xi_{i}\right)$ & Transaction costs incurred by $R S P_{i}$ in transacting with the agent \\
\hline$\hat{c}_{i}\left(s_{i}, \varsigma_{i}\right)$ & Transaction costs incurred by the agent in transacting with $R S P_{i}$ \\
\hline$c_{j}\left(s_{j}, \eta_{j}\right)$ & Transaction costs incurred by the agent in transacting with $R S D_{j}$ \\
\hline$\hat{c}_{j}\left(s_{j}, \theta_{j}\right)$ & Unit transaction cost incurred by $R S D_{j}$ in transacting with the agent \\
\hline$\rho_{1 i}$ & Selling price set by $R S P_{i}$ \\
\hline$\rho_{2 j}$ & Selling price set by the agent in transacting with $R S D_{j}$ \\
\hline$\rho_{3 j}$ & Reserve price set by $R S D_{j}$ \\
\hline$\rho_{3}$ & Vector of the reserve prices with component: $\rho_{31}, \ldots, \rho_{3 n}$ \\
\hline$d_{j}\left(\rho_{3 j}\right)$ & Demand function of $R S D_{j}$ \\
\hline$\varepsilon_{i}$ & $\begin{array}{l}\text { Random variable which represents the uncertainty regarding the service costs of } R S P_{i} \\
\text { with zero mean and variance } \sigma_{p f i}^{2}\end{array}$ \\
\hline$\xi_{i}$ & $\begin{array}{l}\text { Random variable which represents the uncertainty regarding the transaction costs of } \\
R S P_{i} \text { with zero mean and variance } \sigma_{p c i}^{2}\end{array}$ \\
\hline$\varsigma_{i}$ & $\begin{array}{l}\text { Random variable which represents the uncertainty regarding the transaction costs } \\
\text { incurred by the agent in transacting with } R S P_{i} \text { with zero mean and variance } \sigma_{a p i}^{2}\end{array}$ \\
\hline$\eta_{j}$ & $\begin{array}{l}\text { Random variable which represents the uncertainty regarding the transaction costs } \\
\text { incurred by the agent in transacting with } R S D_{j} \text { with zero mean and variance } \sigma_{a d j}^{2}\end{array}$ \\
\hline$\theta_{j}$ & $\begin{array}{l}\text { Random variable which represents the uncertainty regarding the transaction costs } \\
\text { incurred by } R S D_{j} \text { in transacting with the agent with zero mean and variance } \sigma_{d c j}^{2}\end{array}$ \\
\hline
\end{tabular}

In the light of the analyses above, the profit of $R S P_{i}, P_{1 i}$, can be expressed as follows: 


$$
P_{1 i}=\rho_{1 i} s_{i}-f_{i}\left(s_{i}, \varepsilon_{i}\right)-c_{i}\left(s_{i}, \xi_{i}\right)
$$

There are three common models that were used to model risk aversion: mean-variance utility function (Markowitz, 1952), Value at Risk (VaR) criterion and Conditional Value-at-risk (CVaR) criterion (Madadi, 2014). But the latter two are suitable for handling rare but long disruptions (Madadi, 2014). Therefore, we model risk aversion by adopting the mean-variance approach in this paper. Then the utility of $R S P_{i}, U_{1 i}$, can be written as follows:

$$
U_{1 i}=\rho_{1 i} s_{i}-E\left[f_{i}\left(s_{i}, \varepsilon_{i}\right)\right]-E\left[c_{i}\left(s_{i}, \xi_{i}\right)\right]-\beta_{i}\left(\operatorname{Var}\left[f_{i}\left(s_{i}, \varepsilon_{i}\right)\right]+\operatorname{Var}\left[c_{i}\left(s_{i}, \xi_{i}\right)\right]\right),
$$

where $E[\cdot]$ and $\operatorname{Var}[\cdot]$ denote the expectation and variance of the cost function, respectively, and $\beta_{i} \geq 0$ reflects the risk aversion attitude of $R S P_{i}$ towards uncertainty. The larger the $\beta_{i}$, the more conservative the $R S P_{i}$ 's behavior.

Each $R S P_{i}$ is faced, due to technological limitations, with a maximum service capacity, so that

$$
0 \leq s_{i} \leq \bar{s}_{i}
$$

Then the maximum utility of $R S P_{i}$ can be expressed as:

$$
\begin{aligned}
& \operatorname{Max} \rho_{1 i} s_{i}-E\left[f_{i}\left(s_{i}, \varepsilon_{i}\right)\right]-E\left[c_{i}\left(s_{i}, \xi_{i}\right)\right]-\beta_{i}\left(\operatorname{Var}\left[f_{i}\left(s_{i}, \varepsilon_{i}\right)\right]+\operatorname{Var}\left[c_{i}\left(s_{i}, \xi_{i}\right)\right]\right), \\
& \text { subject to } 0 \leq s_{i} \leq \bar{s}_{i}
\end{aligned}
$$

Assuming that the service cost functions and transaction cost functions are continuously differentiable and convex. Let $K_{i}^{1} \equiv\left\{s_{i} \in S^{1} \mid s_{i} \in R_{+}^{m}\right.$, and (3) holds $\}$ denotes the feasible set corresponding to $R S P_{i}$, and define $K^{1} \equiv \prod_{i=1}^{m} K_{i}^{1}$. Then the optimality conditions for all RSPs can be described simultaneously using the following VI (Nagurney et al., 2007):

$$
\sum_{i=1}^{m}\left(\frac{\partial E\left[f_{i}\left(s_{i}^{*}, \varepsilon_{i}\right)\right]}{\partial s_{i}}+\frac{\partial E\left[c_{i}\left(s_{i}^{*}, \xi_{i}\right)\right]}{\partial s_{i}}+\beta_{i}\left(\frac{\partial \operatorname{Var}\left[f_{i}\left(s_{i}^{*}, \varepsilon_{i}\right)\right]}{\partial s_{i}}+\frac{\partial \operatorname{Var}\left[c_{i}\left(s_{i}^{*}, \xi_{i}\right)\right]}{\partial s_{i}}\right)-\rho_{1 i}^{*}\right) \times\left(s_{i}-s_{i}^{*}\right) \geq 0, \forall s_{i} \in K^{1}
$$

where the optimality solution is marked with an asterisk.

\subsubsection{The behavior of the agent}

The agent is involved in transaction with both RSPs and RSDs. Since the services cannot be stored by the agent, it is sensible to as sume that the total quantity of services sold by the agent is equal to the total quantity of services that it purchased from the RSPs. This as sumption can be expressed as the following equation:

$$
\sum_{i=1}^{m} s_{i}=\sum_{j=1}^{n} s_{j}
$$

Then the profit function of the agent, $P_{2}$, can be expressed as follows:

$$
P_{2}=\sum_{j=1}^{n}\left(\rho_{2 j} s_{j}-c_{j}\left(s_{j}, \eta_{j}\right)\right)-\sum_{i=1}^{m}\left(\hat{c}_{i}\left(s_{i}, \varsigma_{i}\right)+\rho_{1 i} s_{i}\right) \text {. }
$$

We have assumed that the agent seeks to maximize its profit. Thus the maximum utility of the agent can be expressed as:

$$
\operatorname{Max} \sum_{j=1}^{n}\left(\rho_{2 j} s_{j}-E\left[c_{j}\left(s_{j}, \eta_{j}\right)\right]\right)-\sum_{i=1}^{m}\left(E\left[\hat{c}_{i}\left(s_{i}, \varsigma_{i}\right)\right]+\rho_{1 i} s_{i}\right)-\beta_{a}\left(\sum_{j=1}^{n} \operatorname{Var}\left[c_{j}\left(s_{j}, \eta_{j}\right)\right]+\sum_{i=1}^{m} \operatorname{Var}\left[\hat{c}_{i}\left(s_{i}, \varsigma_{i}\right)\right]\right),
$$

where $\beta_{a} \geq 0$ represents the degree of risk aversion of the agent. 
Let $K^{2}$ denotes the feasible set corresponding to the agent, and let $\gamma$ denotes the Lagrange multiplier associated with constraint (6). Define $K^{2} \equiv\left\{\left(s_{i}, s_{j}, \gamma\right) \in\left(S^{1}, S^{2}, \gamma\right) \mid\left(s_{i}, s_{j}, \gamma\right) \in R_{+}^{m+n+1}\right.$, and (3) holds, $\left.\forall i, j.\right\}$. Assume that the transaction cost functions are continuously differentiable and convex. Then the optimality conditions for the agent can be expressed as the following VI (Nagurney et al., 2007):

$$
\begin{aligned}
& \sum_{j=1}^{n}\left(\frac{\partial E\left[c_{j}\left(s_{j}^{*}, \eta_{j}\right)\right]}{\partial s_{j}}+\beta_{a} \frac{\partial \operatorname{Var}\left[c_{j}\left(s_{j}^{*}, \eta_{j}\right)\right]}{\partial s_{j}}-\rho_{2 j}^{*}-\gamma^{*}\right) \times\left(s_{j}-s_{j}^{*}\right) \\
& +\sum_{i=1}^{m}\left(\frac{\partial E\left[\hat{c}_{i}\left(s_{i}^{*}, s_{i}\right)\right]}{\partial s_{i}}+\beta_{a} \frac{\partial \operatorname{Var}\left[\hat{c}_{i}\left(s_{i}^{*}, s_{i}\right)\right]}{\partial s_{i}}+\rho_{1 i}^{*}+\gamma^{*}\right) \times\left(s_{i}-s_{i}^{*}\right) \\
& +\left(\sum_{j=1}^{n} s_{j}^{*}-\sum_{i=1}^{m} s_{i}^{*}\right) \times\left(\gamma-\gamma^{*}\right) \geq 0, \forall\left(s_{i}, s_{j}, \gamma\right) \in K^{2} .
\end{aligned}
$$

\subsubsection{The behavior of the RSDs}

The main driving force of a service-oriented network is users' demand for services. As mentioned above, it is assumed that each RSD has an elastic demand for services. Generally, price is an important influence factor of demand since it tends to dominate all other considerations in market transactions. Therefore, we consider that the RSDs' demand for services depends on the reverse price which is the highest price he/she can afford. Assume the demand function of $R S D_{j}$ is of the following form:

$$
d_{j}\left(\rho_{3 j}\right)=a-\omega \rho_{3 j},
$$

where $a$ embodies the effects of all factors other than price on demand and $\omega$ denotes the sensitivity of demand to price. Obviously, the demand function is strictly monotone decreasing and continuously differentiable.

In this paper, we only consider RSDs' utilities from trading with the agent. $R S D_{j}$ 's utility is represented by the margin between his/her reserve payment and actual payment. The larger the margin, the more the $R S D_{j}$ 's utility. Thus the maximum utility of $R S D_{j}$ can be written as follows:

$$
\operatorname{Max} \rho_{3 j} s_{j}-\rho_{2 j} s_{j}-E\left[\hat{c}_{j}\left(s_{j}, \theta_{j}\right)\right]-\beta_{j} \operatorname{Var}\left[\hat{c}_{j}\left(s_{j}, \theta_{j}\right)\right] .
$$

In an equilibrium state, the quantity of services sold by the agent to a RSD should equal to the corresponding demand. Then the equilibrium condition associated with the transaction between the agent and $R S D_{j}$ can be expressed as follows:

$$
d_{j}\left(\rho_{3 j}^{*}\right)\left\{\begin{array}{ll}
=s_{j}^{*} & \text { if } \rho_{3 j}^{*}>0 \\
\leq s_{j}^{*} & \text { if } \rho_{3 j}^{*}=0
\end{array},\right.
$$

As stated in condition (12), in an equilibrium state, if the equilibrium price that $R S D_{j}$ is willing to pay for the services is positive, then the demand of $R S D_{j}$ will be precisely equal to the quantity of services sold by the agent. If the equilibrium price is zero, then the quantity of services sold by the agent may exceed the actual demand.

Let $G\left(\rho_{3 j}\right)=s_{j}-d_{j}\left(\rho_{3 j}\right)$, then Eq. (12) can be rewritten as the following linear complementarity proble m (LCP): determine $\rho_{3 j}^{*} \in R_{+}^{n}$ such that

$$
\left\{\begin{array}{l}
G\left(\rho_{3 j}^{*}\right) \in R_{+}^{n} \\
G\left(\rho_{3 j}^{*}\right) \rho_{3 j}^{*}=0
\end{array}\right.
$$

Since every LCP is a VI problem (Harker and Pang, 1990), the Eq. (12) can be formulated by VI. Thus the optimality conditions for all RSDs can be formu lated simultaneously with the following VI in vector field (Nagurney et 
al., 2007)

$$
\begin{aligned}
& K^{3}=\left\{\left(s_{j}, \rho_{3 j}\right) \in\left(S^{2}, \rho_{3}\right) \mid\left(s_{j}, \rho_{3 j}\right) \in R_{+}^{2 n}, \forall j .\right\}, \\
& \sum_{j=1}^{n}\left(\rho_{2 j}^{*}+\frac{\partial E\left[\hat{c}_{j}\left(s_{j}^{*}, \theta_{j}\right)\right]}{\partial s_{j}}+\beta_{j} \frac{\partial \operatorname{Var}\left[\hat{c}_{j}\left(s_{j}^{*}, \theta_{j}\right)\right]}{\partial s_{j}}-\rho_{3 j}\right) \times\left(s_{j}-s_{j}^{*}\right) \\
& +\sum_{j=1}^{n}\left(s_{j}^{*}-d_{j}\left(\rho_{3 j}^{*}\right)\right) \times\left(\rho_{3 j}-\rho_{3 j}^{*}\right) \geq 0, \forall\left(s_{j}, \rho_{3 j}\right) \in K^{3}
\end{aligned}
$$

\section{The equilibrium conditions of CMfgS}

In equilibrium state, the optimality conditions for all the RSPs, the optimality conditions for the agent and the optimality conditions for all the RSDs must be simultaneously satisfied so that no decision-maker wants to alter his/her decisions.

Definition 1. The equilibrium of CMfgS is one that the flows of services between the tires of the supernetwork model coincide and the flows satisfy the sum of the conditions (5), (9) and (14).

Theorem 1. The equilibrium conditions governing the supernetwork model are equivalent to the solution of the VI problem given by: determine $\left(s_{i}^{*}, s_{j}^{*}, \rho_{3 j}^{*}, \gamma^{*}\right) \in K$ satisfying

$$
\begin{aligned}
& \sum_{i=1}^{m}\left(\frac{\partial E\left[f_{i}\left(s_{i}^{*}, \varepsilon_{i}\right)\right]}{\partial s_{i}}+\frac{\partial E\left[c_{i}\left(s_{i}^{*}, \xi_{i}\right)\right]}{\partial s_{i}}+\beta_{i}\left(\frac{\partial \operatorname{Var}\left[f_{i}\left(s_{i}^{*}, \varepsilon_{i}\right)\right]}{\partial s_{i}}+\frac{\partial \operatorname{Var}\left[c_{i}\left(s_{i}^{*}, \xi_{i}\right)\right]}{\partial s_{i}}\right)\right. \\
& \left.+\frac{\partial E\left[\hat{c}_{i}\left(s_{i}^{*}, s_{i}\right)\right]}{\partial s_{i}}+\beta_{a} \frac{\partial \operatorname{Var}\left[\hat{c}_{i}\left(s_{i}^{*}, s_{i}\right)\right]}{\partial s_{i}}+\gamma^{*}\right) \times\left(s_{i}-s_{i}^{*}\right) \\
& +\sum_{j=1}^{n}\left(\frac{\partial E\left[c_{j}\left(s_{j}^{*}, \eta_{j}\right)\right]}{\partial s_{j}}+\beta_{a} \frac{\partial \operatorname{Var}\left[c_{j}\left(s_{j}^{*}, \eta_{j}\right)\right]}{\partial s_{j}}\right. \\
& \left.+\frac{\partial E\left[\hat{c}_{j}\left(s_{j}^{*}, \theta_{j}\right)\right]}{\partial s_{j}}+\beta_{j} \frac{\partial \operatorname{Var}\left[\hat{c}_{j}\left(s_{j}^{*}, \theta_{j}\right)\right]}{\partial s_{j}}-\gamma^{*}-\rho_{3 j}\right) \times\left(s_{j}-s_{j}^{*}\right) \\
& +\sum_{j=1}^{n}\left(s_{j}^{*}-d_{j}\left(\rho_{3 j}^{*}\right)\right) \times\left(\rho_{3 j}-\rho_{3 j}^{*}\right)+\left(\sum_{j=1}^{n} s_{j}^{*}-\sum_{i=1}^{m} s_{i}^{*}\right) \times\left(\gamma-\gamma^{*}\right) \geq 0, \forall\left(s_{i}, s_{j}, \rho_{3 j}, \gamma\right) \in K .
\end{aligned}
$$

where $K \equiv\left\{\left(s_{i}, s_{j}, \rho_{3 j}, \gamma\right) \in\left(S^{1}, S^{2}, \rho_{3}, \gamma\right) \mid\left(s_{i}, s_{j}, \rho_{3 j}, \gamma\right) \in R_{+}^{m+2 n+1}\right.$, and (3) holds, $\left.\forall i, j.\right\}$.

Proof. We first prove that the equilibrium described in Definition 1 is consistent with the solution of VI (15). Indeed, summing conditions (5), (9) and (14), after simplifications, yields VI (15).

Then we verify the converse, that is, the solution to VI (15) satisfies the sum of conditions (5), (9) and (14). We add the term $\left(\rho_{1 i}^{*}-\rho_{1 i}^{*}\right)$ to the first set of brackets of Eq. (15), and add the term $\left(\rho_{2 i}^{*}-\rho_{2 i}^{*}\right)$ to the second set of brackets. These "terms" don't change the value of Eq. (15) since they equal to zero. Then Eq. (15) can be converted into the sum of conditions (5), (9) and (14). The proof is completed.

$s_{i}$ is bounded since it is subject to the constraint of service capacity (i.e., Eq. (3)). We also impose weak constraints to other variables, then the bounded feasible set $K_{b}$ can be written as:

$$
K_{b} \equiv\left\{\left(s_{i}, s_{j}, \rho_{3 j}, \gamma\right) \in\left(S^{1}, S^{2}, \rho_{3}, \gamma\right) \mid\left(s_{i}, s_{j}, \rho_{3 j}, \gamma\right) \in R_{+}^{m+2 n+1}, 0 \leq s_{i} \leq \bar{s}_{i}, 0 \leq s_{j} \leq \bar{s}_{j}, 0 \leq \rho_{3 j} \leq \bar{\rho}_{3 j}, 0 \leq \gamma \leq \bar{\gamma}, \forall i, j .\right\},
$$

where $\bar{s}_{j}, \bar{\rho}_{3 j}, \quad \bar{\gamma}$ are the upper bounds of $s_{j}, \rho_{3 j}, \quad \gamma$, respectively. Hence, the feasible set $K_{b}$ is compact. The Eq. (16) is reasonable from an economic perspective since the quantity of services and reserve prices must be positive and vary in limited ranges. We can set an upper bound large enough for each of the variable to ensure that the feasible set is 
compact.

For convenience, Eq. (15) can be expressed by the following standard form: determine $X^{*} \in K_{b}$, such that

$$
\left\langle F\left(X^{*}\right), X-X^{*}\right\rangle \geq 0, \forall X \in K_{b},
$$

where $\langle\cdot, \cdot\rangle$ denotes inner product operation, and the specific components of $F(X)$ are given by the respective functional terms preceding the multiplication operators in Eq. (15), and $X \equiv\left(S^{1}, S^{2}, \rho_{3}, \gamma\right)$.

Since we have assumed that all the cost functions and the demand functions are continuously differentiable, according to Brouwer's fixed point theorem (Border, 1989), there is at least one solution for (17). Furthermore, assuming that $F(X)$ is strictly monotonous, then the equilibrium solution is unique (Nagurney et al., 2002).

\section{Influences of risk attitude and cost uncertainty on equilibrium of $\mathrm{CMfgS}$}

To investigate the impacts of risk attitude and cost uncertainty on the equilibrium of CMfg S, we conducted a numeric analysis in this study. Since there are several variables in the model, it is unnecessary to show all the results in this paper. Hence, our investigation focused on the following two aspects: the influences of risk attitude variation on the equilibrium solutions and the impacts of the degree of transaction cost uncertainty on the equilibrium solutions.

We constructed a numerical analysis model with two RSPs and two RSDs. The cost functions of the RSPs were given by:

$$
\begin{aligned}
& f_{1}\left(s_{1}, \varepsilon_{1}\right)=0.5 s_{1}^{2}+\left(2+\varepsilon_{1}\right) s_{1}+10, \quad f_{2}\left(s_{2}, \varepsilon_{2}\right)=0.4 s_{2}^{2}+\left(3+\varepsilon_{2}\right) s_{2}+8, \\
& c_{1}\left(s_{1}, \xi_{1}\right)=\left(10+\xi_{1}\right) s_{1}, \quad c_{2}\left(s_{2}, \xi_{2}\right)=\left(15+\xi_{2}\right) s_{2},
\end{aligned}
$$

and the cost functions of the agent were given by:

$$
c_{1}\left(s_{1}, \eta_{1}\right)=\left(8+\eta_{1}\right) s_{1}, \quad c_{2}\left(s_{2}, \eta_{2}\right)=\left(9+\eta_{2}\right) s_{2}, \quad \hat{c}_{1}\left(s_{1}, \varsigma_{1}\right)=\left(8+\varsigma_{1}\right) s_{1}, \quad \hat{c}_{2}\left(s_{2}, \varsigma_{2}\right)=\left(12+\varsigma_{2}\right) s_{2} .
$$

The demand functions and cost functions of the RSDs were given by:

$$
d_{1}=150-2 \rho_{31}, \quad d_{2}=120-1.5 \rho_{32}, \quad \hat{c}_{1}\left(s_{1}, \theta_{1}\right)=\left(10+\theta_{1}\right) s_{1}, \quad \hat{c}_{2}\left(s_{2}, \theta_{2}\right)=\left(9+\theta_{2}\right) s_{2} .
$$

The upper bounds and lower bounds of all the decision variables were set to 200 and 0 , respectively. These random variables were assumed to satisfy normal distribution and were given by: $\varepsilon_{i} \sim N(0,0.22), \xi_{i} \sim N(0,0.2)$, $\varsigma_{j} \sim N(0,0.15), \eta_{j} \sim N(0,0.1), \theta_{j} \sim N(0,0.25), i=1,2, j=1,2$.

The Euler method (Nagurney et al., 2007) was employed in this paper to address Eq. (17). The convergence criterion utilized was that the difference in the values between two successive iterations no more than $10^{-3}$. The sequence $\left\{\alpha_{\tau}\right\}$ in the Euler method was set to $\{1,1 / 2,1 / 2,1 / 3,1 / 3,1 / 3, \ldots\}$. The algorith $m$ was initialized by a group of random solutions.

\subsection{Influe nces of de cision-makers' risk attitude on equilibrium solutions}

The risk aversion attitude was represented by $\beta_{i}, \beta_{j}, i=1,2, j=1,2$ and $\beta_{a}$ in this study. The $R S P_{i}$ is risk neutral when $\beta_{i}=0$ and absolute risk averse when $\beta_{i}=1$. And the situation is same for $R S D_{j}$ and the agent. To fully reflect the impact of risk attitude on equilibrium of CMfgS, the combination of different decision-makers' risk attitude was considered in this study. Since the two RSPs (RSDs) have similar behavior, we only analyzed the effect of RSP's $\left(\mathrm{RSD}_{1}\right.$ 's) risk attitude variation on equilibrium solutions.

\subsubsection{Influe nces of RSPs' risk attitude}

Let $\beta_{11}$ vary from 0 to 1 at intervals of 0.1 and other parameters are set as follows: $\beta_{12}=0, \beta_{a}=0, \beta_{31}=1, \beta_{32}=0$. We 
will summarize our findings.

Fig. 2 shows the variation of equilibrium prices and equilibrium quantity of services. We can see that all the equilibrium prices go up with the rise of $\beta_{11}$ and all the equilibrium quantity of services fall with $\beta_{11}$ except $s_{12}$. The results indicate that the change in a RSP's risk attitude has an impact on all the decision-makers' behavior. The higher the degree of risk aversion of a RSP, the higher the service prices, the lesser the quantity of services provided by the RSP, and the more the quantity of services provided by other RSPs. The reason is that the risk loss increases with the quantity of services, thus a conservative RSP tends to raise the price to decrease the trading volume. Benefiting from the rise of $\mathrm{RSP}_{1}$ 's price, the demand for $\mathrm{RSP}_{2}$ 's services increases. To equate demand and supply, $\mathrm{RSP}_{2}$ raises price too.

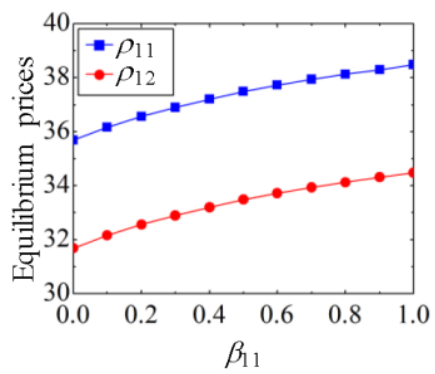

(a)

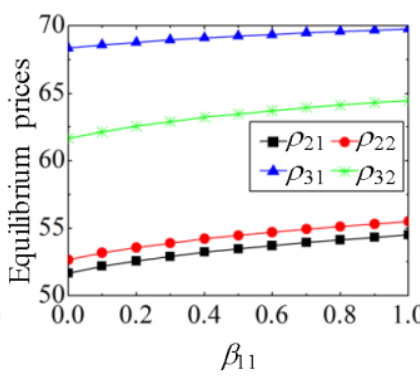

(b)

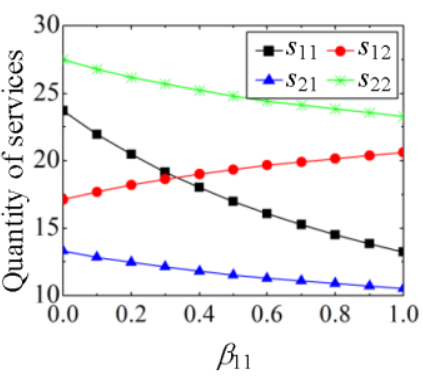

(c)

Fig. 2 Changes of equilibrium solutions as $\mathrm{RSP}_{1}$ 's risk attitude varies.

\subsubsection{Influe nces of the agent's risk attitude}

To analyze the impact of the agent's risk attitude on the equilibrium solutions, we let $\beta_{a}$ vary from 0 to 1 at intervals of 0.1 and other parameters are set as follows: $\beta_{11}=1, \beta_{12}=0, \beta_{31}=1, \beta_{32}=0$. The results are depicted in Fig. 3 .

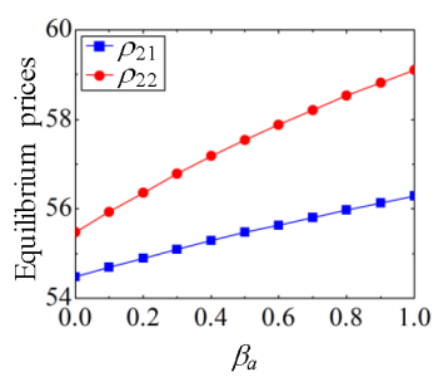

(a)

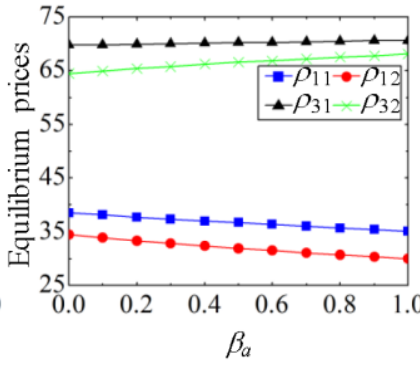

(b)

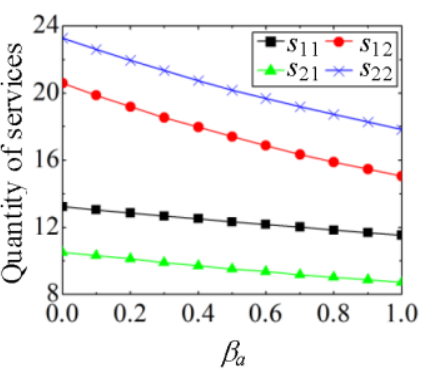

(c)

Fig. 3 Changes of equilibrium solutions as the agent's risk attitude varies.

As shown in Fig. 3, an increase in $\beta_{a}$ leads to decreases in $s_{11}, s_{12}, s_{21}, s_{22}, \rho_{11}$ and $\rho_{12}$ but increases in $\rho_{21}, \rho_{22}, \rho_{31}$ and $\rho_{32}$. Our results show that the rise in the degree of the agent's risk aversion causes a decrease in all the equilibrium quantity of services. A probable explanation is that in an environment with uncertainty, the more conservative the agent is, the more possible it tends to raise the selling price to mitigate risk, which leads to the decrease in RSDs' demand. As a result, the RSPs have to reduce the prices in order to reap additional sales.

\subsubsection{Influences of RSDs' risk attitude}

Let $\beta_{31}$ vary from 0 to 1 at intervals of 0.1 and other parameters are set as follows: $\beta_{11}=1, \beta_{12}=0, \beta_{a}=0, \beta_{32}=0$. The results are portrayed in Fig. 4.

Fig. 4 reveals that an increase in $\beta_{31}$ leads to rises in $\rho_{31}$ and $s_{22}$ but decreases in $\rho_{11}, \rho_{12}, \rho_{21}, \rho_{22}, s_{11}, s_{12}$ and $s_{21}$. An interesting phenomenon is that a risk averse RSD is willing to pay more for the needed services. Our finding is consistent with Ashgarizadeh and Murthy's conclusion which states that a more risk averse customer is willing to pay a higher price for service contract (Ashgarizadeh and Murthy, 2000). While a risk neutral RSD would lower his/her reserve price to purchase more services when other RSDs raise their reserve prices. 


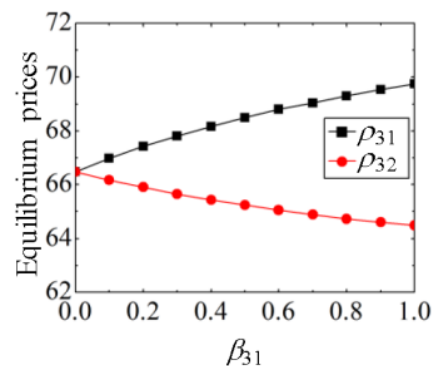

(a)

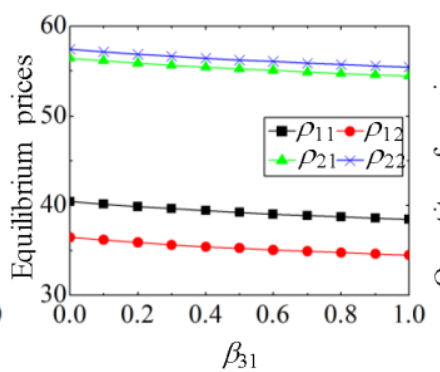

(b)

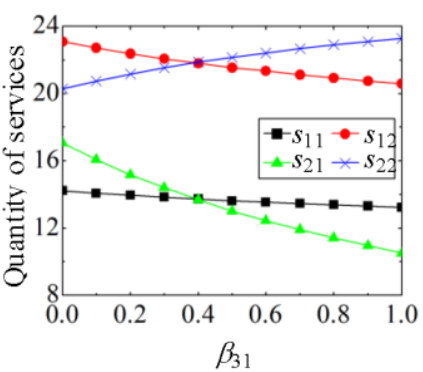

(c)

Fig. 4 Changes of equilibrium solutions as $\mathrm{RSD}_{1}$ 's risk attitude varies.

\subsection{Influences of cost uncertainty on equilibrium solutions}

The degree of uncertainty is expressed usually in terms of a variance or a standard deviation (Chun et al., 2000). To explore whether the degree of cost uncertainty has an obvious influence on equilibrium of CMfgS, we analyzed the impacts of variance change (VC) of decision-makers' cost distribution on the equilibrium solutions. Since the model consists of several random variables, it is unlikely to assess the independent influence of each of them. Hence, we let $\sigma_{p f 1}^{2}=\sigma_{p f 2}^{2}=\sigma_{p f}^{2}, \quad \sigma_{p c 1}^{2}=\sigma_{p c 2}^{2}=\sigma_{p c}^{2}, \quad \sigma_{a p 1}^{2}=\sigma_{a p 2}^{2}=\sigma_{a p}^{2}, \quad \sigma_{a d 1}^{2}=\sigma_{a d 2}^{2}=\sigma_{a d}^{2}$ and $\sigma_{d c 1}^{2}=\sigma_{d c 2}^{2}=\sigma_{d c}^{2}$.

\subsubsection{Influences of VC of RSPs' cost distribution}

We first analyzed the impacts of VC of the RSPs' cost distribution. Since the service cost functions and the transaction cost functions are similar, we only focused on transaction cost uncertainty and let $\sigma_{p c}^{2}$ vary from 0 to 0.5 at intervals of 0.1 . Other parameters are set as follows: $\beta_{11}=1, \beta_{12}=0, \beta_{a}=0, \beta_{31}=1, \beta_{32}=0$. Fig. 5 illustrates the effects of variation of $\sigma_{p c}^{2}$ on the equilibrium solutions.

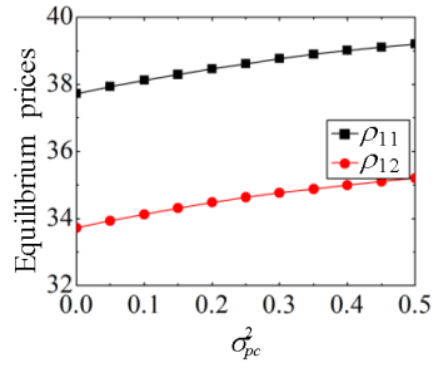

(a)

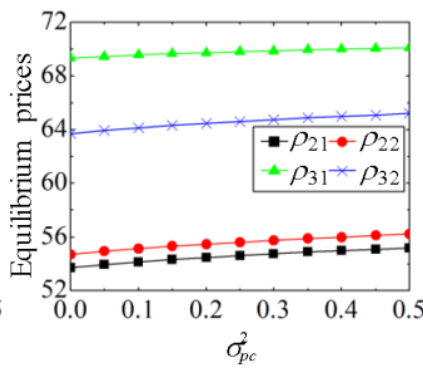

(b)

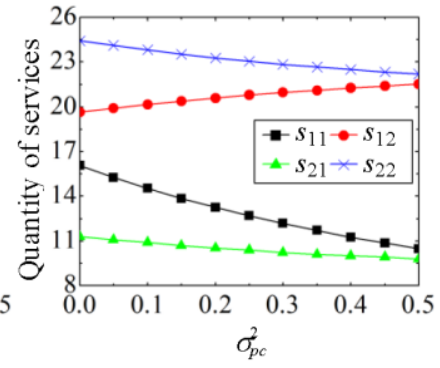

(c)

Fig. 5 Changes of equilibrium solutions as the variance of RSPs' cost distribution varies.

According to Fig. 5, a rise in $\sigma_{p c}^{2}$ leads to decreases in $s_{11}, s_{12}$ and $s_{22}$ but an increase in $s_{12}$. And all the equilibrium prices increase with $\sigma_{p c}^{2}$. The observations imply that the variation in quantity of services provided by a RSP depends on his/her risk attitude under the impact of VC of cost distribution. A risk neutral RSP could sell more services when $\sigma_{p c}^{2}$ is relative large but the opposite occurs for risk averse RSPs. Affected by the rise of the RSPs' prices, the agent's and the RSDs' prices all increase.

\subsubsection{Influe nces of VC of the agent's cost distribution}

Let $\sigma_{a d}^{2}$ vary from 0 to 0.5 at intervals of 0.1 . Other parameters are set as follows: $\beta_{11}=1, \beta_{12}=0, \beta_{a}=1, \beta_{31}=1$, $\beta_{32}=0$. Fig. 6 presents the effects of variation of $\sigma_{a d}^{2}$ on the equilibrium solutions.

From Fig. 6 we can see that the results are very similar to those shown in Fig. 3, which suggest that a rise in either the degree of the agent's risk aversion attitude or its' cost uncertainty has adverse influences on total trading volume. The agent plays a crucial role in $\mathrm{CMfgS}$, and a risk neutral agent is conducive to facilitating service transaction. The 
most interesting aspect of this graph is that $\rho_{21}$ drops down first and then rises up. A probable explanation is that lowering price to reap more sales is a suitable strategy for the agent when the RSDs are risk averse and the cost uncertainty is low. Since $\sigma_{a p}^{2}$ has an impact similar to that of $\sigma_{a d}^{2}$, its results will not be presented in this study.

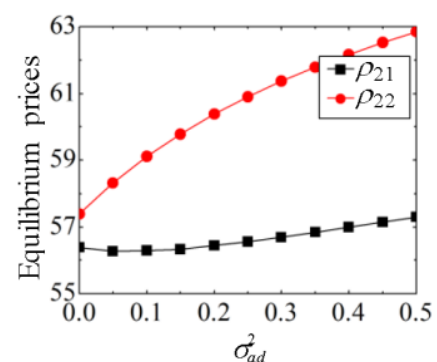

(a)

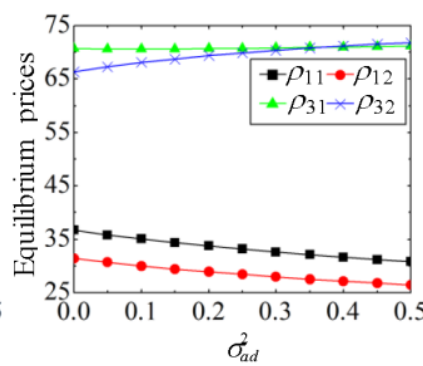

(b)

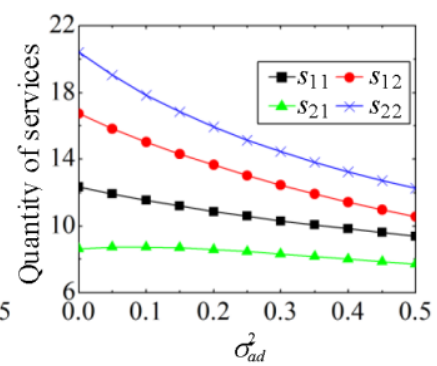

(c)

Fig. 6 Changes of equilibrium solutions as the variance of the agent's cost distribution varies.

\subsubsection{Influe nces of VC of RSDs' cost distribution}

Let $\sigma_{d c}^{2}$ vary from 0 to 0.5 at intervals of 0.1 . Other parameters are set as follows: $\beta_{11}=1, \beta_{12}=0, \beta_{a}=0, \beta_{31}=1$, $\beta_{32}=0$. The results are depicted in Fig. 7 .

As shown in Fig. 7, $\rho_{31}$ and $s_{22}$ increase with the rise of $\sigma_{d c}^{2}$, but $\rho_{11}, \rho_{12}, \rho_{21}, \rho_{22}, s_{11}, s_{12}$ and $s_{21}$ all fall with it. The results are similar to those in Fig. 4, which means that risk attitude variation and VC of cost distribution have analogous impacts on RSDs' behavior.

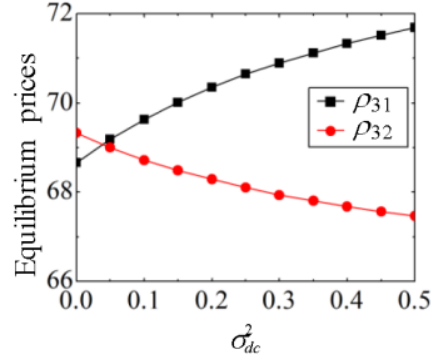

(a)

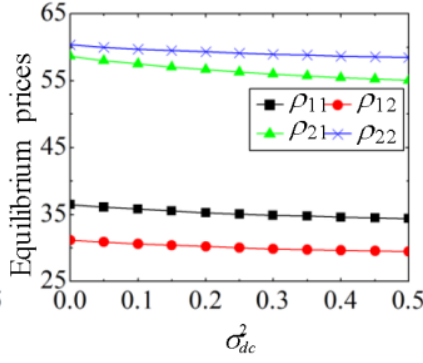

(b)

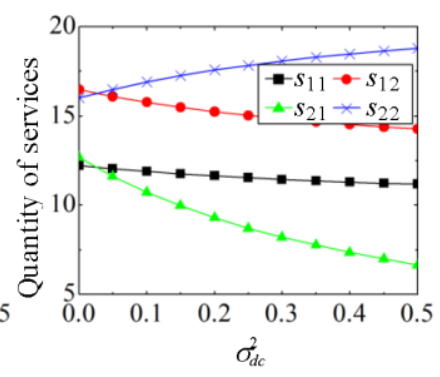

(c)

Fig. 7 Changes of equilibrium solutions as the variance of RSDs' cost distribution varies.

\section{Conclusions}

This study focuses on the equilibrium problem of $\mathrm{CMfgS}$. We modeled the $\mathrm{CMfgS}$ with a three-tier supernetwork. In our analysis, the optimal behavior of the decision-makers consisting of RSPs, the agent and RSDs were modeled, and the uncertainty of service costs and transaction costs were considered in the model. Based on VI theory, we proposed the equilibrium conditions of $\mathrm{CMfgS}$. Specifically, we incorporated the impacts of risk attitude and cost uncertainty into the model to provide insights for deeply understanding the decision-makers' transaction behavior. The influences of the decision-makers' risk attitude and the VC of participants' cost distribution on the equilibrium solutions are investigated through numeric analysis. We summarize some important findings as follows: (1) A change in a decision-maker's risk attitude or variance of cost distribution has an impact on all the participants' behavior. (2) A risk averse RSP (or agent) tends to raise selling price to mitigate risk, and a risk averse RSD is willing to pay more for the needed services, which is consistent with Ashgarizadeh and Murthy's conclusion (Ashgarizadeh and Murthy, 2000). (3) A risk neutral RSP could sell more services when the variance of cost distribution is relative large but the opposite occurs for risk averse RSPs. (4) Risk attitude variation and VC of cost distribution have analogous impacts on RSDs' behavior.

In our study, the demand functions are linear in prices, which cannot cover all the possible situations. An extension could be to model RSDs' behavor with other types of demand, like constant elasticity demand and stochastic demand. 
Our work can also be extended by addressing the decision-makers' risk preference with different models such as Conditional-Value-at-Risk (CVaR) criterion. Despite the limitations, this research extends our knowledge of equilibrium in $\mathrm{CMfgS}$ and will serve as a base for future studies.

\section{Acknowledgments}

This research was partially supported by the National Science-Technology Support Plan Project Grant No. 2015BAF32B03.

\section{References}

Anselmi, J., Ardagna, D. and Passacantando, M., Generalized nash equilibria for saas/paas clouds, European Journal of Operational Research, Vol. 236, No. 1 (2014), pp. 326-339.

Ardagna, D., Pan icucci, B. and Passacantando, M., Generalized Nash equilibria for the service provisioning problem in cloud systems, IEEE Transactions on Services Computing, Vol. 6, No. 4 (2013), pp. 429-442.

Ashgarizadeh, E., and Murthy, D. N. P., Service contracts: A stochastic model, Mathematical and Co mputer Modelling, No. 31 (2000), pp. 11-20.

Askar, S.S., The impact of cost uncertainty on Cournot oligopoly game with concave demand function, Applied Mathematics and Computation, Vol. 232 (2014), pp. 144-149.

Border, K.C., Fixed point theorems with applications to economics and game theory (1989), pp. 28, Cambridge University.

Brown, A.O. and Tang, C.S., The impact of alternative performance measures on single-period inventory policy, Journal of Industrial and Management Optimization, Vol. 2, No. 3 (2006), pp. 297-318.

Caliskan-Demirag, O., Chen, Y.F. and Li, J., Customer and retailer rebates under risk aversion, International Journal of Production Economics, Vol. 133, No. 2 (2011), pp. 736-750.

Cheng, H., Bertrand vs. Cournot equilibriu m with risk averse firms and cost uncertainty, Economic Theory, Vol. 20 , No. 3 (2002), pp. 555-577.

Cheng, Y., Zhao, D., Hu, A. R., Luo, Y. L., Tao, F. and Zhang, L., Multi-view models for cost constitution of cloud service in cloud manufacturing system, International Conference on Computer Science and Education (2011), pp. 225-233.

Chun, M.H., Han S.J., and Tak N.IL., An uncertainty importance measure using a distance metric for the change in a cumulative distribution function, Reliability Engineering and System Safety, No.70 (2000), pp. 313-321.

Harker, P.T. and Pang, J.S., Finite-dimensional variational inequality and nonlinear complementarity problems: a survey of theory, algorithms and applications, Mathematical programming, Vol. 48, No. 1-3 (1990), pp. 161-220.

Krishan, T., Koelemeijer, K. and Rao, R., Consistent assortment provision and service provision in a retail environment, Marketing Science, Vol. 21, No. 1 (2002), pp. 54-73.

Laili, Y., Tao, F., Zhang, L. and Sarker, B.R., A study of optimal allocation of computing resources in cloud manufacturing systems. The International Journal of Advanced Manufacturing Technology, Vol. 63, No.5-8 (2012), pp. 671-690.

Madadi, A., Kurz, M.E., Taaffe, K.M., Sharp, J.L. and Mason, S.J., Supply network design: Risk-averse or risk-neutral? Computers \& Industrial Engineering, Vol. 78 (2014), pp. 55-65.

Markowitz, H., Portfolio selection, The journal of finance, Vol. 7, No. 1 (1952), pp. 77-91.

Mazalov, V., Lukyanenko, A. and Luukkainen, S., Equilibrium in cloud computing market, Performance Evaluation, Vol. 92 (2015), pp. 40-50.

Morgan, J., and O'Donnell, G. E., The cyber physical implementation of cloud manufacturing monitoring systems, 9th CIRP International Conference on Intelligent Computation in Manufacturing Engineering (2015), pp. 29-34.

Nagurney, A., and Wolf, T., A Cournot-Nash-Bertrand game theory model of a service-oriented Internet with price and quality competition among network transport providers, Computational Management Science, Vol. 11, No. 4 (2014), pp. 475-502.

Nagurney, A., Dong, J. and Zhang, D., A supply chain network equilibrium model, Transportation Research Part E: Logistics and Transportation Review, Vol. 38, No. 5 (2002), pp. 281-303. 
Nagurney, A., Liu, Z., Cojocaru, M. G. and Daniele, P., Dynamic electric power supply chains and transportation networks: an evolutionary variational inequality formulation, Transportation Research Part E: Logistics and Transportation Review, Vol. 43, No. 5 (2007), pp. 624-646.

Schweitzer, M.E. and Cachon, G.P., Decision bias in the newsvendor problem with a known demand distribution: Experimental evidence, Management Science, Vol. 46, No. 3 (2000), pp. 404-420.

Shen, Y. and Fan, Y., Cooperative mixed strategy for service selection in service oriented architecture, 2007 IEEE International Conference on Systems, Man, and Cybernetics (2007), pp. 1452-1457.

Sun, T., Li, S. and Zhu, Q., An optimized strategy of service negotiation, 2nd IEEE International Symposium on Service-oriented System Engineering (2006), pp. 210-214.

Tao, F., Cheng, Y., Zhang, L., and Zhao, D., Utility modeling, equilibrium, and coordination of resource service transaction in service-oriented manufacturing system, Proceedings of the Institution of Mechanical Engineers, Part B: Journal of Engineering Manufacture, Vol. 226, No. 6 (2012), pp. 1099-1117.

Wang, X.V. and Xu, X.W., An interoperable solution for cloud manufacturing, Robotics and Computer-Integrated Manufacturing, Vol. 29, No. 4 (2013), pp. 232-247.

Yang, L. and Cai, X., A Game-theoretic analysis to resolve the tussle in cloud storage services, Applied Mathematics \& Information Sciences, Vol. 8, No. 3 (2014), pp. 1361-1367.

Zhang, Z.L., Nabipay, P., Odly zko, A. and Guerin, R., Interactions, competition and innovation in a service-oriented internet: an economic model, IEEE INFOCOM 2010 - IEEE Conference on Computer Communications (2010), pp. 1-5.

Zhen, L., An analytical study on service-oriented manufacturing strategies, International Journal of Production Economics, Vol. 139, No. 1 (2012), pp. 220-228. 